\title{
Peer Review Comments
}

Article: Campbell, L, Chin, K and Stanton, S C E 2016 Initial Evidence that Individuals Form New Relationships with Partners that More Closely Match their Ideal Preferences. Collabra, 2(1): 2, pp. 1-7, DOI: http://dx.doi.org/10.1525/collabra.24

Article type: Original research report

Subject: Psychology

Handling editor: Sam Gosling

Senior editor: Simine Vazire

Article submitted: 12 May 2015

Editor decision: 11 August 2015; 03 November 2015; 06 December 2015

Revision submitted: 25 September 2015; 20 November 2015

Article accepted: 6 December 2015

Article published: 22 January 2016

\section{Responses for Version 1}

Reviewer 1: Paul Eastwick

Affiliation:

Competing Interests Statement: None

Review Completed: 15 July 2015

Recommendation: Resubmit for Review

\section{1) General comments and summary of recommendation:}

Describe your overall impressions and your recommendation, including changes or revisions. Please note that you should pay attention to scientific, methodological, and ethical soundness only, not novelty, topicality, or scope. A checklist of things to you may want to consider is below:

- Are the methodologies used appropriate?

- Are any methodological weaknesses addressed?

- Is all statistical analysis sound?

- Does the conclusion (if present) reflect the argument, is it supported by data/facts?

- Is the article logically structured, succinct, and does the argument flow coherently?

- Are the references adequate and appropriate?

Review by Paul Eastwick

This manuscript has some excellent features. I applaud the way that the authors embarked on this difficult line of work, and I appreciate the high degree of openness and transparency in the authors' reporting. Also, the analyses were conducted and reported very clearly, and I love that the authors have started to address the critical stereotype accuracy confound in this literature. I think this manuscript could make a nice contribution to Collabra. Nevertheless, even though I believe the data certainly merit publication, I have some concerns about the way the prior literature and the current results are being interpreted.

1. A complete depiction of the literature on the predictive validity of ideal partner preferences requires some discussion of methodological choices, and the authors do not get into this nuance. If researchers choose to analyze their data by examining whether the high vs. low value of an ideal for a particular trait predicts the extent to which the trait predicts an outcome (e.g., desire, satisfaction), predictive validity is uniformly poor in attraction contexts and established relationships. (The Li et al., 2013, study is the lone exception, but the very small $\mathrm{N}=8$ targets in that paper renders those effects highly tentative, in my view.) If researchers choose to analyze their data by calculating a pattern of correlations between ideals and a partner's traits across a variety of different traits and use this measure to predict an outcome, predictive validity is weak in attraction contexts but moderate in established relationships.

This study offers a third way of thinking about ideal partner preference predictive validity, but it is quite a different method because there is no DV being predicted (i.e., no desire or satisfaction out- 
come). Thus, the prediction on p. 3 "what people say they want in a future partner should be associated with the actual characteristics of their future partners" wasn't actually predicted or discussed in the ideal standards model literature until now, and it has been examined very rarely in prior work (i.e., only the Burriss and DeBruine papers cited on p. 3). In my mind, this question is closer to questions about assortative mating (i.e., "characteristics of the self are associated with the characteristics of partners") than ideal partner preference predictive validity (i.e., people positively evaluate partners who match rather than mismatch their ideals).

Nevertheless, the correlation between one's ideals and the qualities of partner could indeed be a meaningful/interesting metric, but it is open to many alternative explanations beyond the ones offered here (just as there are many different explanations underlying assortative mating correlations). Perhaps people spend their time in environments where they are likely to encounter people who fit their ideals, so even if you looked at potential partners who were NOT chosen by the participants, the correlations would also be in the $r=.10-.15$ range. Maybe people actively select environments looking for partners who fit their ideals, or maybe people happen to find themselves in certain environments, and those environments shape their ideals.

Indeed, in some speed-dating data that I have on hand, I calculated the same correlations between speed-dating participants' ideals and the qualities of all the people they met at each speed-dating event. The results showed $r=.14$ on average (after controlling for stereotype accuracy), about the same size as the current effects. To be clear: These are not correlations created among speed-dating "matches". These are correlations between participants' ideals and the qualities of ALL 12 people they met at the event. In other words, something about attending a particular speed-dating event like attending an afternoon vs. evening event, or attending an upperclassman vs. underclassman event - is enough to produce correlations of this magnitude. (These correlations are identical if you split the dataset by "yesses" vs. "nos", too.)

(Obviously, the authors aren't obligated to address my data in their revision - I merely note it here to be totally open and to underscore the possibility that these results could emerge through mechanisms other than active partner selection processes.)

Smaller issues:

2. The "key question" that frames the manuscript (i.e., "Do individuals form relationships with others who more closely match their ideal partner preferences") is a bit ambiguous: "more" compared to what exactly? "More" compared to a random other person in the dataset who another participant dated? Yes. "More" compared to another person he/she could have dated but chose not to date? We have no idea.

3. Similarly, the authors cite Elder (1969) and Taylor \& Glenn (1976) as similar approaches on p. 3. Stevens, Owens, and Schaefer (1990) offer an excellent demonstration of how assortative mating is sufficient to explain these effects (i.e., ideal partner preferences do not need to be invoked to explain the data).

4. Although the authors are critical of Asendorpf et al. (2011) for their "exceedingly low" rates of relationship formation (6.6\%), the rates here are quite similar (i.e., 38 out of 450 participants, or 8.4\%). It seems to me that all of these studies (including the current study) are attempting to document a very rare and difficult to capture event.

5. The title of the article is "Initial Evidence for the Predictive Validity of Ideal Partner Preferences in Newly Formed Relationships". But Fletcher et al. (2000) did demonstrate that the pattern correlation between ideals and a partner's traits (similar to the one calculated here) predicted relationship quality outcomes in relationships that were only a few months old. So I'm not sure the authors have documented "initial evidence" for this idea. I think what's really cool and new about this sample is that ideals were assessed before couple members got together; in my view, this is the novel contribution of the current manuscript.

6. Also, do the authors have relationship quality measures? Can they replicate the Fletcher et al. (2000) effects, both with and without subtracting the stereotype accuracy component (which Fletcher did not do)?

7. What are the $\mathrm{df}$ in the multilevel models? And were the traits included as a random factor? It's a little weird how correlations this small can be so significant with an $\mathrm{N}$ of only 38, but this is an element of multilevel modeling that has always eluded me. Nevertheless, the reader may want to know how this is possible. Even though my comments seem critical, the data really are terrific and I would very much like to see them published. I would merely recommend that the authors 
consider shifting some of their language and interpretations of the data to situate this study within the prior literature.

\section{2) Figures/tables/data availability:}

Please comment on the author's use of tables, charts, figures, if relevant. Please acknowledge that adequate underlying data is available to ensure reproducibility (see open data policies per discipline of Collabra here).

Very good.

\section{3) Ethical approval:}

If humans or animals have been used as research subjects, and/or tissue or field sampling, are the necessary statements of ethical approval by a relevant authority present? Where humans have participated in research, informed consent should also be declared.

If not, please detail where you think a further ethics approval/statement/follow-up is required.

No comment.

\section{4) Language:}

Is the text well written and jargon free? Please comment on the quality of English and any need for improvement beyond the scope of this process.

Yes.

Reviewer 2: This reviewer has chosen to remain anonymous

Affiliation:

Competing Interests Statement: I have no competing interests to declare.

Review Completed: 22 June 2015

Recommendation: Revisions Required

\section{1) General comments and summary of recommendation:}

Describe your overall impressions and your recommendation, including changes or revisions. Please note that you should pay attention to scientific, methodological, and ethical soundness only, not novelty, topicality, or scope. A checklist of things to you may want to consider is below:

- Are the methodologies used appropriate?

- Are any methodological weaknesses addressed?

- Is all statistical analysis sound?

- Does the conclusion (if present) reflect the argument, is it supported by data/facts?

- Is the article logically structured, succinct, and does the argument flow coherently?

- Are the references adequate and appropriate?

In the present paper, the authors report evidence that people select their romantic partners according to the ideal partner preferences they state when still being single. To examine this question, the authors conducted a six-month longitudinal study in which they recruited participants when single and asked them for their ideal partner preferences on several traits. If participants entered a romantic relationship during the course of the study, the authors related participants' initially stated preferences to their new romantic partners' self-evaluations on the same traits.

In my opinion, this is really good research and definitely warrants publication in Collabra for three main reasons:

- The research design is great: The longitudinal design allows-for the first time-to actually test whether ideal partner preferences while being single predict the characteristics of a later romantic partner.

- The most important alternative explanations for the obtained effects (i.e., relation due to stereotype accuracy or due to preferring partners that are similar to oneself) are statistically ruled out.

- The paper is generally well written, logically structured, and succinct, and the methods of analysis are sound and appropriate. 
There is one main aspect about the present paper that might be criticized: the sample size $(n=38)$. However, it is really hard to obtain larger sample sizes in the kind of research design that was used in the present paper (i.e., longitudinally following singles and hope that they become involved in a romantic relationship during the course of the study AND then obtain data from their romantic partner). So in my opinion, the sample size should be no hindrance to publication-even more so, as (a) the number of cases entered into the multilevel regressions is much larger (38x38), and (b) the authors openly address the issue of the sample size and take it into account when interpreting their findings.

However, there are some issues the authors should address:

1) Do the results still hold when controlling for both stereotype accuracy and similarity simultaneously (i.e., by entering them into the regressions together)?

2) The authors should mention that their Model 2 (association between new partners' ideal preferences and the original participants' self-evaluations) has some drawbacks in testing their hypothesis: When entering the relationship, the new partners might have adjusted their ideal partner preferences to fit the characteristics of the original participants-which would artificially inflate the investigated association.

3) If the data exist, it would be interesting to know about the stability of the ideal partner preferences over time. Because it only makes sense to use initial partner preferences as predictors of later partner choices if these preferences are stable over time.

4) The demographics suggest that not all examined couples were heterosexual. For comparison with prior findings, it might be interesting to also have the results when including only heterosexual couples in the analyses (as all prior research that found no predictive validity of ideal partner preferences in real-life encounters used heterosexual samples only).

5) In the interpretation of their findings (p. 11 and 12), the authors could make more explicit that it was self-ascribed characteristics of the new partner that were predicted by the ideal partner preferences. This doesn't mean that the new partners actually ARE the way they state to be (which is, however, suggested by the phrases used by the authors: e.g., "[‥] people do appear to enter relationships with others possessing qualities that correspond more closely to their stated preferences when single", p.12; "[‥] individuals form relationships with others who more closely match their ideal partner preferences", p.12).

6) Because the drop-out rate was quite high (of 167 couples only 38 provided partner data), authors might discuss whether there is any chance that there was systematic drop-out of couples in which the partner did NOT match the ideal partner preferences.

7) The authors might contemplate to add some information on statistical power/sample size in multilevel modeling (for readers who are not that familiar with multilevel modeling and who might therefore be misled by the small sample size).

8) If statistical power allows, it would be interesting to examine (a) gender differences, and (b) the influence of relationship duration on the results. For example, one might hypothesize that the predictive validity of ideal partner preferences becomes stronger in longer-lasting relationships (because people might enter short romantic relationships with partners that do not match their preferences, but in the long run probably only the relationships with matching partners will persist).

\section{2) Figures/tables/data availability:}

Please comment on the author's use of tables, charts, figures, if relevant. Please acknowledge that adequate underlying data is available to ensure reproducibility (see open data policies per discipline of Collabra here).

All tables are well-arranged, concise, and easily understood. Each table is warrantable and useful.

The authors state that the data can be obtained from the first author on request.

\section{3) Ethical approval:}

If humans or animals have been used as research subjects, and/or tissue or field sampling, are the necessary statements of ethical approval by a relevant authority present? Where humans have participated in research, informed consent should also be declared.

If not, please detail where you think a further ethics approval/statement/follow-up is required. 
Informed consent is declared and the authors state that the present research was approved by their local ethics board.

\section{4) Language:}

Is the text well written and jargon free? Please comment on the quality of English and any need for improvement beyond the scope of this process.

The language should better be evaluated by a native speaker.

\section{Editor Decision for Version 1}

Editor: Sam Gosling

Affiliation: University of Texas, Austin, USA

Editor decision: Revisions required

Decision date: 11 August 2015

Dear Prof. Lorne Campbell,

After review, we have reached a decision regarding your submission to Collabra, "Initial Evidence for the Predictive Validity of Ideal Partner Preferences in Newly Formed Relationships". Our decision is to request revisions of the manuscript. These revisions may then undergo further peer review prior to acceptance.

The full review information should be included at the bottom of this email. There may also be a copy of the manuscript file with reviewer comments available once you have accessed the submission account. A summary of the requested edits from the editorial team can be found below. Please consider these points and revise the file accordingly:

Editorial Revision Requests:

I write with my decision on your article, "Initial Evidence for the Predictive Validity of Ideal Partner Preferences in Newly Formed Relationships," which you submitted for consideration in COLLBRA.

I now have two excellent reviews of your manuscript from experts in the field. Both experts agree that the topic is important and your questions are interesting. They rightly point to several positive features of the paper including the longitudinal design, assessing ideals partners before relationships were initiated, and the fact that you control for stereotype accuracy and similarity. Both reviewers also explicitly make the point that these data should be published, and I agree with them.

However, the reviews have convinced me that that the analyses do not show exactly what they may appear to show. The difference is one that is subtle (in fact I would have missed it without the experts' input) but important. As Reviewer 1 (Paul Eastwick, who signed his review) notes, there are number of other reasons-other than active partner selection-that could account for similarities in descriptions of ideal partners and new partners' self-descriptions; in light of research showing that people choose to be in places where others share their values (e.g., Motyl et al., 2014, JESP, 51, 1-14), the possibility that people of similar personalities are prevalent in one's surroundings seems quite compelling. The current analyses do not allow you to tell whether the new partner is more or less similar to the participant than their other alternatives. Thus, as Reviewer notes in his 2nd point, "The 'key question' that frames the manuscript (i.e., 'Do individuals form relationships with others who more closely match their ideal partner preferences') is a bit ambiguous: 'more' compared to what exactly? 'More' compared to a random other person in the dataset who another participant dated? Yes. 'More' compared to another person he/she could have dated but chose not to date? We have no idea." As I note above, the difference in interpretation is subtle but it's one that needs to be made clear to the reader and requires a partial reframing of the paper.

Both reviewers also ask whether you have any DVs that could be predicted from the ideal-actual partner relations. Reviewer 2 suggests that relationship longevity might be a good bet and Reviewer 1 asks about relationship quality. Such an analysis would complement the past work on the topic.

Beyond that, the expertise of both reviewers is reflected in their thoughtful and constructive additional suggestions (e.g., to help the readers understand how small samples can render such correlations significant in multi-level modeling designs). I urge you to integrate these ideas into a revision, 
should you choose to undertake one.

To access your submission account, follow the below instructions:

1) login to the journal webpage with username and password

2) click on the submission title

3) click 'Review' menu option

4) download Reviewed file and make revisions based on review feedback

5) upload the edited file

6) Click the 'notify editor' icon and email the confirmation of re-submission and any relevant comments to the journal.

Please ensure that your revised files adhere to our author guidelines, and that the files are fully copyedited/proofed prior to upload. Please also ensure that all copyright permissions have been obtained. This is the last opportunity for major editing, therefore please fully check your file prior to re-submission.

If you have any questions or difficulties during this process, please do contact us.

Please could you have the revisions submitted by Aug 26th 2015. If you cannot make this deadline, please let us know as early as possible.

Kind regards,

Sam Gosling

University of Texas, Austin, USA

gosling@psy.utexas.edu

Collabra

http://www.collabra.org/

@collabraoa

\section{Author's Response to Review Comments for Version 1}

Corresponding Author: Lorne Campbell

Affiliation: The University of Western Ontario

Revision submitted: 25 September 2015

We have uploaded our revised manuscript (with new title) and cover letter. I clicked "submit revision", with no noticeable consequences.

\section{Attached document:}

September 25, 2015

Dear Dr. Sam Gosling,

Thank you for the invitation to revise our manuscript for further consideration for publication in $\mathrm{Col}$ labra. Below we address the two main points you raised, and then discuss the remaining points of the two reviewers. 
1. In your letter you say "However, the reviews have convinced me that that the analyses do not show exactly what they may appear to show. The difference is one that is subtle (in fact I would have missed it without the experts' input) but important. As Reviewer 1 (Paul Eastwick, who signed his review) notes there are number of other reasons-other than active partner selection-that could account for similarities in descriptions of ideal partners and new partners' self-descriptions; in light of research showing that people choose to be in places where others share their values (e.g., Motyl et al., 2014, JESP, 51,1-14), the possibility that people of similar personalities are prevalent in one's surroundings seems quite compelling. The current analyses do not allow you to tell whether the new partner is more or less similar to the participant than their other alternatives. Thus, as Reviewer notes in his 2nd point, "The 'key question' that frames the manuscript (i.e., 'Do individuals form relationships with others who more closely match their ideal partner preferences') is a bit ambiguous: 'more' compared to what exactly? 'More' compared to a random other person in the dataset who another participant dated? Yes. 'More' compared to another person he/she could have dated but chose not to date? We have no idea." As I note above, the difference in interpretation is subtle but it's one that needs to be made clear to the reader and requires a partial reframing of the paper."

We appreciate this discussion of how our results can, and should, be interpreted. In our analyses, the results demonstrate that the within-dyad association between stated ideal preferences when single and the self-perceptions of new partners across 38 traits is positive and statistically significant (even after controlling for partner similarity and stereotype accuracy). The answer to the question 'Do individuals form relationships with others who more closely match their ideal preferences?' is therefore a qualified 'yes' (qualified, for example, by the size of the effect, the traits used, the specific sample used). In this context "more" is compared to "less", and we added this comparison in the manuscript when asking this question. That said, we completely agree that these results do not inform the actual process of mate choice (i.e., how the two people decided to start dating each other, in what context they first met). In the original discussion section we did mention that our results do not inform the process of actual mate choice, but we have now rewritten the discussion section to focus more squarely on what the results suggest, possible interpretations of the results, and potentially fruitful future directions to discover the mechanism(s) underlying the positive associations between ideal mate preferences when single and the self-perceptions of dating partners in newly formed relationships. We have also taken care to remove wording (e.g., "predictive validity of ideal partner preferences") when referring to our results that can possibly imply analyses we did not conduct (i.e., how the correspondence between partner preferences and the self-evaluations of the new partner predicted relationship formation).

2. You also mentioned that both reviewers also ask whether we have any DVs that could be predicted from the ideal-actual partner relations. In the original submission we discussed how we felt we simply did not have enough dyads $(\mathrm{N}=38)$ to conduct between-dyad analyses predicting, for example, relationship satisfaction from the similarity between ideal preferences and the self-perceptions of new partners. We have not conducted any between-dyad analyses with our data, and we are planning a new study to collect a larger sample of dyads to both replicate the effects reported in this manuscript and test additional predictions as suggested by the reviewers. It is our hope to have this data collected within a few years.

\section{Additional Comments from Reviewer 1 (Paul Eastwick)}

Reviewer 1, Paul, discusses how much prior research has typically attempted to predict various outcomes with the correspondence between ideal preferences and the qualities of the potential or actual partner (e.g., attraction to a hypothetical or real prospective partner; relationship satisfaction in an existing relationship). In our research we simply tested whether such correspondence between ideal preferences and the self-reported qualities of partners in newly formed relationships even existed on average given the conclusions of some researchers (including Paul, as the two of us have discussed privately on a few occasions) that individuals may not preferentially pursue partners that more, versus less, closely match their preferences. Many studies ask people to state their ideal partner preferences with the implicit assumption that these preferences drive, in some way, the actual mate choices of these individuals. It seemed important to us, therefore, to determine if individuals do tend to form relationships with individuals that more, versus less, match their stated ideal preferences. Our review of the literature suggests that no research to date has collected data addressing this question with longitudinal data tracking individuals as they transitioned into actual new relationships. Our primary research focus, therefore, was not the degree to which the correspondence between ideals and partner qualities predicted various outcomes (e.g., desire to date someone; happiness in a relationship), but rather if across a sample of newly formed relationships there even 
exists a positive degree of correspondence across a number of traits. As our results suggest, within dyads there is indeed a positive association between ideal preferences stated when single and the self-evaluations of partners in newly formed relationships, and this association holds when controlling for partner similarity and stereotype accuracy. These results perhaps imply that individuals do preferentially pursue others that more closely match own preferences across a number of traits, but our data does not directly test this possibility. We do not know how these relationships formed, or the context in which they formed, but when they formed people were more likely to be with someone with a pattern of self-evaluations across many traits that corresponded more versus less closely with their own preferences.

We appreciate Paul providing some data from his own speed dating studies. There is a lack of detail provided about these analyses that can be expected given it is a review of our work and not a submission of a manuscript to be reviewed itself (e.g., how many items in the ideal preference/selfevaluation scale? Exactly what are all of the items? Were all of the items part of these analyses? Was a similar data analytic approach used? [the reporting of correlations suggests a similar analytic approach to our own was not used, but it is difficult to tell], and how many of people formed actual romantic relationships with someone from the same speed-dating event? etc.). Furthermore, these results are potentially specific to a speed-dating context on a university campus (e.g., all participants being single, of a similar age range, attending the same university, and choosing to sign-up for a speed-dating event with the intent of meeting prospective dating partners) that may not extend to every possible social context where new partners can be found (e.g., grocery store, library, music store, website, a trip made to Paris on a whim, and so on). We do acknowledge in the discussion section, however, that preferences could influence choice of social context, and choice of social context could influence the pool of prospective partners available.

\section{Minor Points}

- We now cite Stevens, Owens and Schaefer (1990) on page 3.

- Paul says that "Although the authors are critical of Asendorpf et al. (2011) for their "exceedingly low" rates of relationship formation (6.6\%), the rates here are quite similar (i.e., 38 out of 450 participants, or $8.4 \%$ ). It seems to me that all of these studies (including the current study) are attempting to document a very rare and difficult to capture event."

- We feel it important to stress that we were not being critical of Asendorpf et al. for the exceedingly low rates of relationship formation in their study, but were rather pointing out that this low rate of relationship formation made it impossible to adequately test with inferential statistics if individuals ended up in relationship with other speed-daters that more versus less matched their ideal preferences. We were stating a fact, not making a value judgment. To make this more clear, however, we have added the italicized text to the following sentence on page 5: "Only Asendorpf et al. (2011) reported the probabilities for various kinds of future contact among study participants, with rates of actual relationship formation being quite low (i.e., $6.6 \%$ at 6 weeks after the speed-dating event, and $4.4 \%$ at 1 year after the speed-dating event), meaning it was not possible to determine if participants entered new relationships with other speed-dating participants that more or less matched their preferences."

- Additionally, it is not correct to say in our study the rate of relationship formation was $8.4 \%$ given that we began with a sample of 450 single participants and removed 24 for the stated reasons; of these 167 participants indicated starting a new relationship; of these 85 provided with the contact information of their new partners, and of these 38 new partners joined the study. So, the difficulty in our study was recruiting new partners to join the study.

- We have changed the title to better reflect the nature of the research: Initial evidence that individuals form new relationships with partners that more (versus less) closely match their ideal preferences.

- Paul said "What are the df in the multilevel models? And were the traits included as a random factor? It's a little weird how correlations this small can be so significant with an N of only 38, but this is an element of multilevel modeling that has always eluded me. Nevertheless, the reader may want to know how this is possible." We have added the degrees of freedom in the note at the bottom of Table 2. We did not present correlations as a measure of correspondence between ideal preferences and self-evaluations of partners; we presented unstandardized regression coefficients from hierarchical linear models. Although there is no standard measure of effect size for hierarchical linear models, we did calculate an approximate effect size $(r)$ and presented these values in the table, but these values do not represent the within-couple correlation between ideal preferences and self-perceptions across the 38 traits. Instead, the unstandardized regres- 
sion coefficients represent this association, with values ranging from .27-.42 in model 1, and .25-.37 in model 2. Furthermore, the analysis is essentially a repeated measure analysis, with 38 pairs of scores (i.e., ideal preferences predicting self-perceptions) across 38 traits, resulting in 1444 data points. The SAS code for the analyses is available on the OSF project site, and it shows that the independent variable (ideal preferences across the 38 traits) was indeed treated as a random factor.

\section{Comments by Reviewer 2}

1. Do the results still hold when controlling for both stereotype accuracy and similarity simultaneously (i.e., by entering them into the regressions together)?

- The models that control for stereotype accuracy contain scores across all of the traits (both ideal preferences and self-evaluations) that have different mean scores subtracted from them. Entering the self-evaluations of the original participant in these models would no longer be estimating (and hence controlling) purely for similarity, making this suggested analysis not feasible.

2. The authors should mention that their Model 2 (association between new partners' ideal preferences and the original participants' self-evaluations) has some drawbacks in testing their hypothesis: When entering the relationship, the new partners might have adjusted their ideal partner preferences to fit the characteristics of the original participants-which would artificially inflate the investigated association.

- We do mention that this model is not the best test of our hypotheses, as suggested, in footnote 2 on page 9.

3. If the data exist, it would be interesting to know about the stability of the ideal partner preferences over time. Because it only makes sense to use initial partner preferences as predictors of later partner choices if these preferences are stable over time.

- This data is not available in the current study.

4. The demographics suggest that not all examined couples were heterosexual. For comparison with prior findings, it might be interesting to also have the results when including only heterosexual couples in the analyses (as all prior research that found no predictive validity of ideal partner preferences in real-life encounters used heterosexual samples only).

- There were four same-sex couples in the data set, and removing them does not alter the results. Given the small number of same-sex couples in our sample, we do not present the results of these analyses in the manuscript.

5. In the interpretation of their findings (p. 11 and 12), the authors could make more explicit that it was self-ascribed characteristics of the new partner that were predicted by the ideal partner preferences. This doesn't mean that the new partners actually ARE the way they state to be (which is, however, suggested by the phrases used by the authors: e.g., "[‥] people do appear to enter relationships with others possessing qualities that correspond more closely to their stated preferences when single", p.12; "[‥] individuals form relationships with others who more closely match their ideal partner preferences", p.12).

- We have made the suggested changes where appropriate (i.e., adding "self-reported" in many instances),

6. Because the drop-out rate was quite high (of 167 couples only 38 provided partner data), authors might discuss whether there is any chance that there was systematic drop-out of couples in which the partner did NOT match the ideal partner preferences.

- These couples did not drop out of the study, but rather many of the original participants did not share their new partner's contact information, and not all new partners that were contacted accepted our invitation to participate in this study.

7. The authors might contemplate to add some information on statistical power/sample size in multilevel modeling (for readers who are not that familiar with multilevel modeling and who might therefore be misled by the small sample size).

- In the discussion section we do mention that the analyses have repeated measures within couples (38 couples with 38 repeated measures, for 1444 data points), but that we did not feel we had enough couples to adequately test between-couple hypotheses (i.e., low power) 
8. If statistical power allows, it would be interesting to examine (a) gender differences, and (b) the influence of relationship duration on the results. For example, one might hypothesize that the predictive validity of ideal partner preferences becomes stronger in longer-lasting relationships (because people might enter short romantic relationships with partners that do not match their preferences, but in the long run probably only the relationships with matching partners will persist).

- These are good ideas, but see the point above about testing between-couple hypotheses

We would like to end by thanking you and the two reviewers for the thoughtful feedback on our manuscript. We do feel that with the suggested edits the manuscript more clearly, and accurately, conveys the results of our research.

Lorne Campbell

Kristi Chin

Sarah C.E. Stanton

\title{
Responses for Version 2
}

\author{
Reviewer 1: Paul Eastwick \\ Affiliation: \\ Competing Interests Statement: None \\ Review Completed: 23 October 2015 \\ Recommendation: Revisions Required
}

\section{1) General comments and summary of recommendation:}

Describe your overall impressions and your recommendation, including changes or revisions. Please note that you should pay attention to scientific, methodological, and ethical soundness only, not novelty, topicality, or scope. A checklist of things to you may want to consider is below:

- Are the methodologies used appropriate?

- Are any methodological weaknesses addressed?

- Is all statistical analysis sound?

- Does the conclusion (if present) reflect the argument, is it supported by data/facts?

- Is the article logically structured, succinct, and does the argument flow coherently?

- Are the references adequate and appropriate?

Although this manuscript has some excellent features, I think the introduction continues to be confusing, especially for readers unfamiliar with this literature. I will offer concrete recommendations, as I think the issues are quite fixable.

The introduction discusses two analysis strategies, each of which has its own conceptual meaning and possible underlying mechanisms. Analysis strategy \#1 involves using the *match* between ideals and a partner's qualities to predict an outcome, addressing the conceptual question "Does the match between ideals and a partner's traits guide romantic evaluations?" Analysis strategy \#2 involves calculating whether the *match* is significantly different from zero, addressing the conceptual question "Do people form relationships with partners who match their ideals more closely than a randomly selected alternative partner (from the same dataset)?" In principle, either or both could produce significant results - they are independent tests with independent meanings. For example, the mechanism suggested by the Motyl paper is applicable to analysis strategy \#2 but not so applicable to analysis strategy \#1.

In the introduction, the Eastwick studies, the Li study, the Fletcher study, and the Todd study use analysis strategy \#1. You can use a linguistic convention along the lines of "Eastwick and Todd say $x$, but Fletcher and Li say $y^{\prime \prime}$ if you want - it is logically sound. (I think that convention misconstrues the strength of the evidence for $x$ vs. $y$, but that's my opinion.)

The Burriss study, the DeBruine study, and the current study use analysis strategy \#2. (Kenrick \& Keefe, Elder, and Taylor \& Glenn, also use something like analysis strategy \#2, albeit indirectly.) So it isn't logically sound to say Eastwick and Todd say x, but Burriss, DeBruine, et al. say y. That convention is very confusing because these studies do not address the same question, yet this is how paragraphs 2 and 3 are set up (i.e., paragraph 3 begins "on the other hand"). You can describe both literatures, but please explain that they address different questions with different plausible underlying mechanisms. It is very 
confusing as currently written.

The confusion emerges again on pp. 5-6. Campbell and Stanton (2014) make a great point that few studies in the Eastwick meta-analysis test analysis strategy \#1 in a context where people are actually forming romantic relationships. But the current study does not address this gap because it tests analysis strategy \#2. The linguistic conventions on pp. 5-6 imply otherwise: Paragraph 5 suggests "There is a gap in the literature that was meta-analyzed", and paragraph 6 suggests "We conducted a study to address it", but the study does not, in fact, address the meta-analytic gap.

There are solutions to all of this: Describe in the introduction the two research questions that correspond to the two analysis strategies. Tell the reader what it could mean if each one were supported vs. unsupported. Then note that you're going to address conceptual question/analysis strategy \#2. And you're doing it in a cool/novel context (newly formed relationships) with measures that are better than anyone else's.

A final point - the addition of "(vs. less)" throughout does not say much - "(vs. less)" is implied by "more". The missing element is "than" - more *than a randomly selected alternative partner from the same dataset*. I know it's clunky - maybe there's a more elegant way to say it - but that's what the test is.

Sorry to be a pain about this - it's awkward because I have outed myself as the reviewer and we all know each other, and I respect the authors and their work a great deal. I am just so concerned about this literature getting muddied, and I do think that everything works beautifully with some additional clarification and a reorganization of the introduction.

\section{2) Figures/tables/data availability:}

Please comment on the author's use of tables, charts, figures, if relevant. Please acknowledge that adequate underlying data is available to ensure reproducibility (see open data policies per discipline of Collabra here).

All ok.

\section{3) Ethical approval:}

If humans or animals have been used as research subjects, and/or tissue or field sampling, are the necessary statements of ethical approval by a relevant authority present? Where humans have participated in research, informed consent should also be declared.

If not, please detail where you think a further ethics approval/statement/follow-up is required.

All ok.

\section{4) Language:}

Is the text well written and jargon free? Please comment on the quality of English and any need for improvement beyond the scope of this process.

All ok.

\section{Editor Decision for Version 2}

Editor: Sam Gosling

Affiliation: University of Texas, Austin, USA

Editor decision: Revisions required

Decision date: 03 November 2015

Dear Lorne,

Sorry for the delay yet again (somehow I didn't get [or see] the notification that the review was ready).

As I noted in my earlier correspondence with you, my sense was that your revised manuscript did not properly address the reviewer's key concern in terms of the framing of your question. I thought the concern was important because it highlighted minor but consequential differences in what your data mean. As I also noted before, I think your data are informative and worthy of publishing even if they address this slightly different question. But it is crucial that the subtle differences in what these findings do and 
do not say are made clear to readers. The reviewer has made another attempt to lay out his point. So I invite you to revise the manuscript in way that is responsive to this way of describing the two questions, taking care to note which of the questions the previous literature do and do not address and which of them your data do and do not address too.

If you believe that the reviewer and I are mistaken about the differences in the two questions, please let me know and we can try again to get to the bottom of this issue.

To access your submission account, follow the below instructions:

1) login to the journal webpage with username and password

2) click on the submission title

3) click 'Review' menu option

4) download Reviewed file and make revisions based on review feedback

5) upload the edited file

6) Click the 'notify editor' icon and email the confirmation of re-submission and any relevant comments to the journal.

Please ensure that your revised files adhere to our author guidelines, and that the files are fully copyedited/ proofed prior to upload. Please also ensure that all copyright permissions have been obtained. This is the last opportunity for major editing;, therefore please fully check your file prior to re-submission.

If you have any questions or difficulties during this process, please do contact us.

Please could you have the revisions submitted by Nov 19. If you cannot make this deadline, please let us know as early as possible.

Kind regards,

Sam Gosling

University of Texas, Austin, USA

samg@austin.utexas.edu

Reviewer A:

Although this manuscript has some excellent features, I think the introduction continues to be confusing, especially for readers unfamiliar with this literature. I will offer concrete recommendations, as I think the issues are quite fixable.

The introduction discusses two analysis strategies, each of which has its own conceptual meaning and possible underlying mechanisms. Analysis strategy \#1 involves using the *match* between ideals and a partner's qualities to predict an outcome, addressing the conceptual question "Does the match between ideals and a partner's traits guide romantic evaluations?" Analysis strategy \#2 involves calculating whether the *match* is significantly different from zero, addressing the conceptual question "Do people form relationships with partners who match their ideals more closely than a randomly selected alternative partner (from the same dataset)?" In principle, either or both could produce significant results - they are independent tests with independent meanings. For example, the mechanism suggested by the Motyl paper is applicable to analysis strategy \#2 but not so applicable to analysis strategy \#1.

In the introduction, the Eastwick studies, the Li study, the Fletcher study, and the Todd study use analysis strategy \#1. You can use a linguistic convention along the lines of "Eastwick and Todd say $x$, but Fletcher and Li say $y^{\prime \prime}$ if you want - it is logically sound. (I think that convention misconstrues the strength of the evidence for $x$ vs. $y$, but that's my opinion.)

The Burriss study, the DeBruine study, and the current study use analysis strategy \#2. (Kenrick \& Keefe, Elder, and Taylor \& Glenn, also use something like analysis strategy \#2, albeit indirectly.) So it isn't logically sound to say Eastwick and Todd say x, but Burriss, DeBruine, et al. say y. That convention is very 
confusing because these studies do not address the same question, yet this is how paragraphs 2 and 3 are set up (i.e., paragraph 3 begins "on the other hand"). You can describe both literatures, but please explain that they address different questions with different plausible underlying mechanisms. It is very confusing as currently written.

The confusion emerges again on pp. 5-6. Campbell and Stanton (2014) make a great point that few studies in the Eastwick meta-analysis test analysis strategy \#1 in a context where people are actually forming romantic relationships. But the current study does not address this gap because it tests analysis strategy \#2. The linguistic conventions on pp. 5-6 imply otherwise: Paragraph 5 suggests "There is a gap in the literature that was meta-analyzed", and paragraph 6 suggests "We conducted a study to address it", but the study does not, in fact, address the meta-analytic gap.

There are solutions to all of this: Describe in the introduction the two research questions that correspond to the two analysis strategies. Tell the reader what it could mean if each one were supported vs. unsupported. Then note that you're going to address conceptual question/analysis strategy \#2. And you're doing it in a cool/novel context (newly formed relationships) with measures that are better than anyone else's.

A final point - the addition of "(vs. less)" throughout does not say much - "(vs. less)" is implied by "more". The missing element is "than" - more *than a randomly selected alternative partner from the same dataset*. I know it's clunky - maybe there's a more elegant way to say it - but that's what the test is.

Sorry to be a pain about this - it's awkward because I have outed myself as the reviewer and we all know each other, and I respect the authors and their work a great deal. I am just so concerned about this literature getting muddied, and I do think that everything works beautifully with some additional clarification and a reorganization of the introduction.

Collabra

http://www.collabra.org/

@collabraoa

\section{Author's Response to Review Comments for Version 2}

Corresponding Author: Lorne Campbell

Affiliation: The University of Western Ontario

Revision submitted: 20 November 2015

Hello Sam. We have uploaded a second revision and cover letter for our ms, now titled "Initial Evidence that Individuals Form New Relationships with Partners that More Closely Match their Ideal Preferences".

Best.

\section{Attached document:}

November 20, 2015

Dear Dr. Sam Gosling,

Thank you for the invitation to revise again our manuscript, now titled "Initial Evidence that Individuals Form New Relationships with Partners that More Closely Match their Ideal Preferences", for further consideration for publication in Collabra.

In our opinion the revised manuscript is sensitive to the suggestions of the reviewer, echoed in your own comments, that the introduction now (a) more carefully distinguishes the two primary approaches for assessing links between ideal preferences and study outcomes used in the existing literature, and (b) clearly specifies up front the approach adopted in our own research. The edits have been made throughout the entire introduction. As one example, here is the new second paragraph of the introduction:

"There are two approaches typically used in this growing body of research. The first approach assesses 
the degree of similarity between, for example, preferences for a given trait and how much a current romantic partner possesses that trait, and if this similarity is statistically different from zero. It is assumed that mate choice was guided in some way by preferences if the degree of similarity assessed is positive and statistically significant. The second approach assesses if the degree of similarity between preferences for a given trait and whether a potential partner possesses that trait predicts outcomes associated with mate choice. It is assumed that if higher vs. lower degrees of similarity predict, for example, romantic interest, then individuals may preferentially pursue potential partners because of a high degree of similarity between this person's qualities and the pursuer's preferences. Both approaches are similar in that they can be used to investigate the role of ideal partner preferences in mate choice. They also differ, however, in that the first approach investigates the degree of similarity between preferences and partner characteristics, whereas the second approach uses this degree of similarity as a predictor of outcomes associated with mate choice; each approach can thus be used to answer different questions about the role ideal preferences and mate choice."

After discussing the existing literature we also clearly point out that the transition into new romantic relationships has not been a context for this research up to this point, meaning that no empirical data currently exists speaking to the different questions that can be addressed by each approach in the relationship formation process. Our research addresses this gap in the literature (i.e., it focuses on the transition into new romantic relationships). In discussing these points and setting up the rationale for our current study, here is (partly) what we say on page 6:

\begin{abstract}
"Regardless of the approach used in the study ideal preferences and mate choice, therefore, the lack of research on the process of relationship formation means that we are not yet able to determine (a) if individuals enter new relationships with others that match their ideal preferences more closely, or (b) if the degree of similarity between one's ideal preferences and the qualities of potential partners predicts relationship formation and development. The present research was designed to address question (a), or to ascertain the degree to which individuals enter relationships with partners who embody their ideal preferences."
\end{abstract}

To summarize our conclusions we say: "The data presented therefore suggests that people do appear to enter relationships with others that self-report possessing qualities that correspond more closely to their stated preferences when single." But we also clearly state that our data do not speak to the actual mate selection process in our sample, and we make suggestions for future research on this topic.

We look forward to your feedback on our revised manuscript.

Lorne Campbell

Kristi Chin

Sarah C.E. Stanton

\title{
Editor Decision for Version 3
}

Editor: Sam Gosling

Affiliation: University of Texas, Austin, USA

Editor decision: Accept Submission

Decision date: 06 December 2015

Dear Prof. Lorne Campbell,

After review, we have reached a decision regarding your submission to Collabra, "Initial Evidence for the Predictive Validity of Ideal Partner Preferences in Newly Formed Relationships", and are happy to accept your submission for publication, pending the completion of copyediting and formatting processes.

As there are no further reviewer revisions to make, you do not have to complete any tasks at this point. The accepted submission will now undergo final copyediting. You will be contacted once this is complete to answer any queries that may have arisen during copyediting and to allow a final chance to edit the files prior to typesetting. If you wish to view your submission during this time, you can log in via the journal website.

The review information should be included in this email. 
Kind regards,

Sam Gosling

University of Texas, Austin, USA

samg@austin.utexas.edu

Collabra

http://www.collabra.org/

@collabraoa 